\title{
A Schistosomiasis Model with Mating Structure
}

\author{
Longxing $\mathrm{Qi}^{1}$ and Jing-an $\mathrm{Cui}^{2}$ \\ ${ }^{1}$ School of Mathematical Sciences, Anhui University, Hefei 230601, China \\ ${ }^{2}$ College of Science, Beijing University of Civil Engineering and Architecture, Beijing 100044, China
}

Correspondence should be addressed to Longxing Qi; qilx@ahu.edu.cn

Received 10 July 2013; Accepted 20 August 2013

Academic Editor: Mark McKibben

Copyright (C) 2013 L. Qi and J.-a. Cui. This is an open access article distributed under the Creative Commons Attribution License, which permits unrestricted use, distribution, and reproduction in any medium, provided the original work is properly cited.

\begin{abstract}
A schistosomiasis model is proposed including single schistosomes, paired schistosomes, snails, and the latent period of infected snails. A reasonable sex ratio of schistosomes and the minimum mating function are considered. A threshold condition determining the stability of the system is given, and the stability of equilibrium for the model is shown. The impact of the latent period of infected snails on schistosomiasis transmission can be found through numerical simulations. Finally, preferable control strategies are obtained by sensitivity analyses. Killing snails may be the preferred control measure. If we choose chemotherapy, we should use some drugs which are sufficient for reducing egg-associated pathology, since paired schistosomes are mostly harmful to definitive hosts.
\end{abstract}

\section{Introduction}

Schistosomiasis is one of the most prevalent parasite diseases. Its transmission needs two hosts: the definitive hosts and the intermediate snail hosts. In definitive hosts, schistosoma has two distinct sexes. Female and male schistosomes pair up they lay eggs that work their way through the intestinal lining or into the ureters and bladder. The eggs themselves are harmless, but in definitive host the body's immune response results in inflammation and eventual damage, as granulomasfibrous lesions-form around the eggs. These eggs pass in urine or feces into fresh water. In infected water, miracidium hatches from egg and infects the intermediate snail hosts. In snail hosts, parasites asexually reproduce. As a parasite in the body of the snail, the miracidium loses its cilia and undergoes a change into a sac-like primary sporocysts [1]. Many secondary sporocysts are formed in the body of the primary sporocysts. They develop for some time and then rupture the wall of the primary sporocyst and become established in the body of the snail. From the walls of the secondary sporocyst a large number of parthenogenetic eggs are formed, and they develop internally into forms known as cercariae. After about four, weeks cercariae rupture the body-wall of the secondary sporocyst, bore out of the snail's body, and enter the water as very active organisms, characterized by two large suckers and a forked tail. In this stage, they are ready to enter a mammalian host. When such a free-living cercaria comes in contact with a susceptible mammal, it penetrate, the skin of the definitive host and transforms into single schistosoma. Here, the parasite's life cycle is completed.

During the asexual phase in the host mollusc, several groups have observed that the miracidia are already sexually differentiated [2]. Furthermore, male miracidia will only give rise to male later forms, whilst female miracidia will only give rise to female later forms. However, no apparent differences have been registered between physiological alterations in snails infected by a male miracidium and those in snails infected by a female miracidium. There are no distinctive qualitative and quantitative differences in snail host response to infection with a single miracidium and in snail host reaction to infection with numerous miracidia secured by single or multiple exposures [3]. From the above statement, we know that the mating of male schistosoma and female schistosoma in definitive hosts is very important to the transmission of schistosomiasis. Hence, we differentiate female and male parasites only in definitive hosts in this paper.

In the literature, two-sex problems have been studied by many authors [4-6]. All of these models are established for human population diseases, such as sexually transmitted diseases. They constructed pair-formation models and studied 
the existence, uniqueness and the stabilities of exponential solutions. In $[5,6]$ authors put forward three forms of pairformation functions which are also called mating functions: the harmonic mean function, the geometric mean function and the minimum function. All of these three functions can be applied to two-sex models.

As for schistosomiasis, there are few papers considering two-sex problems and mating interactions [4, 7-9]. In [9] Xu et al. have proposed a multi-strain schistosome model with mating structure. Their goal is to infer the impact of drug treatment on the maintenance of schistosome genetic diversity. However, the model of $\mathrm{Xu}$ et al. ignores several stages of the parasite's complex life cycle. They consider only the adult parasite populations. Furthermore, in their model they assume that the recruitment rate of single adult parasites at time $t$ depends instantaneously on the total number of parasite pairs at time $t$. Subsequently, Castillo-Chavez et al. [4] have relaxed this assumption on the parasite's life history by introducing a time delay. The time delay accounts for the average time that must elapse between two adult generations. Their model is in the following:

$$
\begin{gathered}
\frac{d m}{d t}=k S_{m}(\tau) p(t-\tau)-\left(\mu_{m}+\frac{\sigma}{\theta}\right) m(t)-\varphi(m(t), f(t)), \\
\frac{d f}{d t}=k S_{f}(\tau) p(t-\tau)-\left(\mu_{f}+\frac{\sigma}{\theta}\right) f(t)-\varphi(m(t), f(t)), \\
\frac{d p}{d t}=\varphi(m(t), f(t))-\left(\mu_{p}+\frac{\sigma}{\theta}\right) p(t) .
\end{gathered}
$$

Here $m(t)$ and $f(t)$ represent the densities of single male schistosoma and single female schistosoma, respectively, and $p(t)$ is the density of pairs. The formation of schistosome pairs is described by a mating function $\varphi(m(t), f(t)) . k$ is half of the birth rate of a pair per capita. Note that the ratio of male to female offspring is assumed to be $1: 1$ in their model. $\mu_{m}, \mu_{f}$, and $\mu_{p}$ are the per capita death rates of single male schistosoma, single female schistosoma, and schistosoma in a mated pair, respectively. $\sigma$ is the rate of the chemotherapeutic treatment. $\theta$ denotes the drug resistance of that parasite strain. $S_{m}(\tau)$ and $S_{f}(\tau)$ are functions which keep track of the parasite's survival probabilities in various (nonadult) stages of the life cycle. The dynamical behaviors of their model in [4] are not qualitatively different from those derived from an earlier model in [9], although [9] ignores the impact of time delays associated with the multiple stages in parasite's life cycle. The results of [4] imply that higher treatment rate can allow for coexistence between susceptible and resistant parasite strains. Both in $[4,9]$, the harmonic mean function is chosen as the mating function $\varphi(m(t), f(t))$.

As we know, in the two models of $[4,9]$, the ratio of male to female offspring is assumed to be $1: 1$. In fact, some experiments have shown that the number of male schistosomes is bigger than that of female [10-16]. In [10] authors found the phenomena of the natural male bias by experimental observations on the sex ratio of adult schistosoma mansoni. In [12] the sex ratio of female schistosoma to male schistosoma is $1: 1.81$. In [16], authors reported that the male-biased sex ratio is 2.36 males to 1 female in Kenya. In two experiments of [14], the sex ratios of female schistosoma to male schistosoma are $(0.89 \pm 0.03): 1$ and $(0.98 \pm 0.03): 1$, respectively. These results tend to male-biased sex ratio. In [15] the sex ratio of female schistosoma to male schistosoma is $1: 1.38$. In artificial experiments of [13], there are 9543 males and 6597 females in 16140 mature schistosoma japonicum; the ratio of female to male is $1: 1.45$. In [11] authors also support male-biased sex ratio. All of these results imply that the sex ratio is not $1: 1$. Furthermore, Mao [13] also denote that the assumption of the $50: 50$ sex ratio in previous schistosomiasis models does not accord with the result of experiments. Hence, in this paper, we consider reasonably that the number of male offspring is bigger than that of female in our model.

On the other hand, the two models in $[4,9]$ do not include the snail dynamics. In reality, from the life cycle of schistosoma, it is easy to see that the parasite offspring is produced directly by infected snails but not by paired parasites. Nåsell [17] also thought that the number of male and female schistosoma is proportional to the number of infected snails. This implies that the snail dynamics may influence the transmission of schistosomiasis. Hence, it is necessary to add snails to the model. In addition, from the life history of schistosoma, we know that the production of parasite offspring needs about four weeks after the snail hosts are infected by miracidia $[4,9]$. Motivated by $[4,9,13,17]$ we established a new model including a more reasonable sex ratio of schistosoma, snail dynamics, the latent period of infected snails, and the mating structure of schistosoma. Here, we take the minimum function as mating function. In this paper, our purpose is to study the dynamics of single schistosoma, pairs, and snails and to put forward preferable control strategies.

Our paper is organized as follows. In Section 2, we establish a mathematical model with single schistosoma, pairs and snails. For convenience of analysis, we first study the model without time delay and define the basic reproductive number. And then the stabilities of the disease-free and endemic equilibria are obtained in Section 3. In Section 4, the stability of the delayed model is investigated. In Section 5, computational simulations and sensitivity analysis are performed, and we give preferable control strategies.

\section{Mathematical Model}

Considering the mating structure of parasites and snail dynamics, we propose a model with state variables $m(t)$, $f(t), p(t), s(t)$, and $i(t)$, where $m(t), f(t)$, and $p(t)$ represent the densities of single male schistosoma, single female schistosoma and pairs, respectively; $s(t)$ and $i(t)$ represent the number of susceptible and infectious snail hosts, respectively. The basic assumptions are as follows.

(i) Since the single male and female schistosoma are from the cercaria produced by infected snails, in $[17,18]$ authors consider that the number of male and female schistosoma is proportional to the number of infected snails. According to $[17,18]$ we also assume that the recruitment rate of single male and female schistosoma is proportional to the number of infected snail hosts, that is, the recruitment rates of single 
male and female worms are $k_{m} i(t)$ and $k_{f} i(t)$, respectively. Following papers [10-16], we assume $k_{m} \geq k_{f}$.

(ii) Natural death rates for single male, single female, and pair worms per capita are $\mu_{m}, \mu_{f}$, and $\mu_{p}$, respectively. In reality, pairs of schistosoma may live for a few years while single parasites may only live for a few weeks [9]. Therefore, we assume $\mu_{p}<\mu_{m}, \mu_{f}$. As for the investigation of the death rate of single male and female schistosoma, there are many results. In the experiment of [12], the livability of single male parasites is stronger than that of single female parasites, which implies that single female parasites have a higher natural death rate than single male parasites. In [19], the author found that single male schistosoma is larger more muscular. May and Woolhouse [20] and Tchuente et al. [21] also found that the growth of single female schistosoma is restrained when it is not paired up. Standen [22] deduced that single female schistosoma is incapable of going against the blood stream but single male schistosoma can. These results imply that the livability of single male parasites is stronger than that of single female parasites. Cornford and Huot [23] and Cornford and Fitzpatrick $[24,25]$ had shown that single female schistosoma has less glucose than single male schistosoma. B. G. Atkinson and K. H. Atkinson [26] and Davis et al. [27] found that single female schistosoma has less actin than single male schistosoma. These indicate that single female parasite is not easy to survive. In addition, Mao [13] also reported that the survival rate of single male schistosoma is not lower than that of single female schistosoma. All of the above results imply $\mu_{m} \leq \mu_{f}$. Hence, we assume $\mu_{p}<\mu_{m} \leq \mu_{f}$.

(iii) The mating function $\varphi(m(t), f(t))$ takes the minimum function $\varphi(m(t), f(t))=\rho \min (m(t), f(t))$ in which $\rho$ represents the effective mating rate $[5,6]$.

(iv) The parameter $\Lambda_{s}$ is the recruitment rate of snail hosts. $\mu_{s}$ is per capita natural death rate of snail hosts. $\alpha_{s}$ is the disease-induced death rate of snail hosts. The transmission rate from pairs to susceptible snails is a constant $\xi$.

(v) $\tau$ is the latent period of infected snails, and $e^{-\left(\mu_{s}+\alpha_{s}\right) \tau}$ represents the survival rate of infected snails in the latent period.

(vi) We suppose that infected snails do not recover from schistosomiasis as their life spans are short.

(vii) All parameters are assumed nonnegative in reality.

Then, we have a model with the following form:

$$
\begin{aligned}
& \frac{d m}{d t}=k_{m} i(t-\tau) e^{-\left(\mu_{s}+\alpha_{s}\right) \tau}-\mu_{m} m(t)-\varphi(m(t), f(t)), \\
& \frac{d f}{d t}=k_{f} i(t-\tau) e^{-\left(\mu_{s}+\alpha_{s}\right) \tau}-\mu_{f} f(t)-\varphi(m(t), f(t)), \\
& \frac{d p}{d t}=\varphi(m(t), f(t))-\mu_{p} p(t), \\
& \frac{d s}{d t}=\Lambda_{s}-\mu_{s} s(t)-\xi p(t) s(t), \\
& \frac{d i}{d t}=\xi p(t) s(t)-\left(\mu_{s}+\alpha_{s}\right) i(t) .
\end{aligned}
$$

For convenience of the stability analysis of (2), we first study the case when $\tau=0$, that is,

$$
\begin{aligned}
\frac{d m}{d t} & =k_{m} i(t)-\mu_{m} m(t)-\varphi(m(t), f(t)), \\
\frac{d f}{d t} & =k_{f} i(t)-\mu_{f} f(t)-\varphi(m(t), f(t)), \\
\frac{d p}{d t} & =\varphi(m(t), f(t))-\mu_{p} p(t), \\
\frac{d s}{d t} & =\Lambda_{s}-\mu_{s} s(t)-\xi p(t) s(t), \\
\frac{d i}{d t} & =\xi p(t) s(t)-\left(\mu_{s}+\alpha_{s}\right) i(t) .
\end{aligned}
$$

In the current paper, we take the mating function $\varphi(m(t)$, $f(t))=\rho \min (m(t), f(t))[5,6]$. The function is linear on each of the following two sets:

$$
\begin{gathered}
K_{f}=\left\{(m, f, p, s, i) \in R^{5} \mid f \geq m \geq 0,\right. \\
\left.p \geq 0, s \geq 0, i \geq 0,0 \leq s+i \leq \frac{\Lambda_{s}}{\mu_{s}}\right\}, \\
K_{m}=\left\{(m, f, p, s, i) \in R^{5} \mid m \geq f \geq 0,\right. \\
\left.p \geq 0, s \geq 0, i \geq 0,0 \leq s+i \leq \frac{\Lambda_{s}}{\mu_{s}}\right\} .
\end{gathered}
$$

In general, none of these sets is positively invariant for (3) with $\varphi(m(t), f(t))=\rho \min (m(t), f(t))$. But under the conditions

$$
k_{m} \geq k_{f}, \quad \mu_{p}<\mu_{m} \leq \mu_{f},
$$

the set $K_{m}$ is positively invariant. Thus, the model (3) with an initial surplus of males becomes the so-called female dominance model on the set $K_{m}$ and the system (3) can be rewritten in the following form:

$$
\begin{aligned}
\frac{d m}{d t} & =k_{m} i(t)-\mu_{m} m(t)-\rho f(t), \\
\frac{d f}{d t} & =k_{f} i(t)-\mu_{f} f(t)-\rho f(t), \\
\frac{d p}{d t} & =\rho f(t)-\mu_{p} p(t), \\
\frac{d s}{d t} & =\Lambda_{s}-\mu_{s} s(t)-\xi p(t) s(t), \\
\frac{d i}{d t} & =\xi p(t) s(t)-\left(\mu_{s}+\alpha_{s}\right) i(t) .
\end{aligned}
$$

Using the standard method, it is easy to see that the disease-free equilibrium $E_{0}=\left(0,0,0, \Lambda_{s} / \mu_{s}, 0\right)$ always exists. According to the calculational method of the basic 
reproductive number [28], the basic reproduction number for model (6) is

$$
R_{0}=\sqrt[3]{\frac{\xi \rho \Lambda_{s} k_{f}}{\mu_{s}\left(\mu_{s}+\alpha_{s}\right) \mu_{p}\left(\mu_{f}+\rho\right)}} .
$$

The following section shows that the basic reproductive number $R_{0}$ provides a threshold condition for parasite extinction in (6).

\section{Stability Analysis of the System (6)}

In this section, we will analyze the stability of model (6). The stability of the disease-free equilibrium determines whether the disease will be prevalent in an uninfected population. The following result shows that the parasites will go extinct if $R_{0}<$ 1 .

Theorem 1. The disease-free equilibrium $E_{0}$ of the system (6) is locally asymptotically stable if $R_{0}<1$ and unstable if $R_{0}>1$.

Proof. The Jacobian matrix for system (6) is given by

$$
J=\left(\begin{array}{ccccc}
-\mu_{m} & -\rho & 0 & 0 & k_{m} \\
0 & -\left(\mu_{f}+\rho\right) & 0 & 0 & k_{f} \\
0 & \rho & -\mu_{p} & 0 & 0 \\
0 & 0 & -\xi_{s}(t) & -\mu_{s}-\xi_{p}(t) & 0 \\
0 & 0 & \xi s(t) & \xi_{p}(t) & -\left(\mu_{s}+\alpha_{s}\right)
\end{array}\right) .
$$

Thus, the eigenvalues of $E_{0}$ are $-\mu_{s},-\mu_{m}$, and the roots of the equation are

$$
\lambda^{3}+a_{1} \lambda^{2}+a_{2} \lambda+a_{3}=0
$$

where

$$
\begin{aligned}
& a_{1}=\left(\mu_{s}+\alpha_{s}\right)+\mu_{p}+\left(\mu_{f}+\rho\right)>0, \\
& a_{2}=\left(\mu_{s}+\alpha_{s}\right) \mu_{p}+\left(\mu_{s}+\alpha_{s}\right)\left(\mu_{f}+\rho\right)+\mu_{p}\left(\mu_{f}+\rho\right)>0, \\
& a_{3}=\left(\mu_{s}+\alpha_{s}\right) \mu_{p}\left(\mu_{f}+\rho\right)-\frac{\xi \rho \Lambda_{s} k_{f}}{\mu_{s}} .
\end{aligned}
$$

Note that $a_{3}>0$ if and only if $R_{0}<1$.

By the Routh-Hurwitz criterion, we know

$$
\begin{aligned}
& H_{1}=a_{1}>0, \\
& H_{2}=\left|\begin{array}{cc}
a_{1} & a_{3} \\
1 & a_{2}
\end{array}\right|>0, \\
& H_{3}=\left|\begin{array}{ccc}
a_{1} & a_{3} & 0 \\
1 & a_{2} & 0 \\
0 & a_{1} & a_{3}
\end{array}\right|=a_{3} H_{2},
\end{aligned}
$$

which implies that all the eigenvalues of $E_{0}$ have negative real parts if and only if $R_{0}<1$. It follows that the disease-free equilibrium $E_{0}$ is locally asymptotically stable if $R_{0}<1$ and unstable if $R_{0}>1$.
Note that if $R_{0}>1,(6)$ has a unique endemic equilibrium $E^{*}=\left(m^{*}, f^{*}, p^{*}, s^{*}, i^{*}\right)$, where

$$
\begin{aligned}
s^{*} & =\frac{\left(\mu_{s}+\alpha_{s}\right) \mu_{p}\left(\mu_{f}+\rho\right)}{\xi \rho k_{f}}, \\
p^{*} & =\frac{\Lambda_{s} \rho k_{f}}{\left(\mu_{s}+\alpha_{s}\right) \mu_{p}\left(\mu_{f}+\rho\right)}-\frac{\mu_{s}}{\xi}=\frac{\mu_{s}}{\xi}\left(R_{0}^{3}-1\right), \\
f^{*} & =\frac{\mu_{p}}{\rho} p^{*}, \\
i^{*} & =\frac{\mu_{p}\left(\mu_{f}+\rho\right)}{\rho k_{f}} p^{*}, \\
m^{*} & =\frac{k_{m}\left(\mu_{f}+\rho\right)-\rho k_{f}}{k_{f} \mu_{m}} \frac{\mu_{p}}{\rho} p^{*} .
\end{aligned}
$$

Similarly, using the Routh-Hurtwitz criterion we can obtain the stability of the endemic equilibrium $E^{*}$.

Theorem 2. The endemic equilibrium $E^{*}$ of the system (6) is locally asymptotically stable if $R_{0}>1$.

\section{Stability Analysis of the System (2)}

In this section, we analyze the stability of model (2). We recall it with the following form:

$$
\begin{aligned}
\frac{d m}{d t} & =k_{m} i(t-\tau) e^{-\left(\mu_{s}+\alpha_{s}\right) \tau}-\mu_{m} m(t)-\rho f(t), \\
\frac{d f}{d t} & =k_{f} i(t-\tau) e^{-\left(\mu_{s}+\alpha_{s}\right) \tau}-\mu_{f} f(t)-\rho f(t), \\
\frac{d p}{d t} & =\rho f(t)-\mu_{p} p(t), \\
\frac{d s}{d t} & =\Lambda_{s}-\mu_{s} s(t)-\xi_{p}(t) s(t), \\
\frac{d i}{d t} & =\xi p(t) s(t)-\left(\mu_{s}+\alpha_{s}\right) i(t) .
\end{aligned}
$$

Define

$$
\widetilde{R}_{0}=\frac{\xi \rho \Lambda_{s} k_{f} e^{-\left(\mu_{s}+\alpha_{s}\right) \tau}}{\mu_{s}\left(\mu_{s}+\alpha_{s}\right) \mu_{p}\left(\mu_{f}+\rho\right)} .
$$

We can obtain that the disease-free equilibrium $\widetilde{E}_{0}=$ $\left(0,0,0, \Lambda_{s} / \mu_{s}, 0\right)$ always exists and if $\widetilde{R}_{0}>1$ there exists the endemic equilibrium $\widetilde{E}^{*}=\left(\widetilde{m}^{*}, \widetilde{f}^{*}, \widetilde{p}^{*}, \widetilde{s}^{*}, \widetilde{i}^{*}\right)$, where

$$
\begin{aligned}
& \widetilde{s}^{*}=\frac{\left(\mu_{s}+\alpha_{s}\right) \mu_{p}\left(\mu_{f}+\rho\right)}{\xi \rho k_{f} e^{-\left(\mu_{s}+\alpha_{s}\right) \tau}}, \\
& \widetilde{p}^{*}=\frac{\Lambda_{s} \rho k_{f} e^{-\left(\mu_{s}+\alpha_{s}\right) \tau}}{\left(\mu_{s}+\alpha_{s}\right) \mu_{p}\left(\mu_{f}+\rho\right)}-\frac{\mu_{s}}{\xi}=\frac{\mu_{s}}{\xi}\left(\widetilde{R}_{0}-1\right), \\
& \widetilde{f}^{*}=\frac{\mu_{p}}{\rho} \widetilde{p}^{*},
\end{aligned}
$$




$$
\begin{gathered}
\widetilde{i}^{*}=\frac{\mu_{p}\left(\mu_{f}+\rho\right)}{\rho k_{f} e^{-\left(\mu_{s}+\alpha_{s}\right) \tau}} \widetilde{p}^{*}, \\
\widetilde{m}^{*}=\frac{k_{m}\left(\mu_{f}+\rho\right)-\rho k_{f}}{k_{f} \mu_{m}} \frac{\mu_{p}}{\rho} \widetilde{p}^{*} .
\end{gathered}
$$

$$
|J-\lambda I|=\left|\begin{array}{ccccc}
-\left(\mu_{m}+\frac{\sigma}{\theta}\right) & -\rho & 0 & 0 & k_{m} e^{-\left(\mu_{s}+\alpha_{s}\right) \tau} e^{-\lambda} \\
0 & -\left(\mu_{f}+\rho+\frac{\sigma}{\theta}\right) & 0 & 0 & k_{f} e^{-\left(\mu_{s}+\alpha_{s}\right) \tau} e^{-\lambda} \\
0 & \rho & -\left(\mu_{p}+\frac{\sigma}{\theta}\right) & 0 & 0 \\
0 & 0 & -\xi s(t) & -\mu_{s}-\xi p(t) & 0 \\
0 & 0 & \xi s(t) & \xi p(t) & -\left(\mu_{s}+\alpha_{s}\right)
\end{array}\right| .
$$

Thus, the eigenvalues of $\widetilde{E}_{0}$ are $-\mu_{s},-\mu_{m}$ and the roots of the equation are

$$
\lambda^{3}+\tilde{a}_{1} \lambda^{2}+\tilde{a}_{2} \lambda+\tilde{a}_{3}=0
$$

where

$$
\begin{aligned}
& \tilde{a}_{1}=\left(\mu_{s}+\alpha_{s}\right)+\mu_{p}+\left(\mu_{f}+\rho\right)>0, \\
& \tilde{a}_{2}=\left(\mu_{s}+\alpha_{s}\right) \mu_{p}+\left(\mu_{s}+\alpha_{s}\right)\left(\mu_{f}+\rho\right)+\mu_{p}\left(\mu_{f}+\rho\right)>0, \\
& \tilde{a}_{3}=\left(\mu_{s}+\alpha_{s}\right) \mu_{p}\left(\mu_{f}+\rho\right)-\frac{\xi \rho \Lambda_{s} k_{f}}{\mu_{s}} e^{-\left(\mu_{s}+\alpha_{s}\right) \tau} e^{-\lambda} .
\end{aligned}
$$

If $\lambda \geq 0$ and $\tau>0,0<e^{-\lambda} \leq 1$, and then

$$
\widetilde{a}_{3} \geq\left(\mu_{s}+\alpha_{s}\right) \mu_{p}\left(\mu_{f}+\rho\right)-\frac{\xi \rho \Lambda_{s} k_{f}}{\mu_{s}} e^{-\left(\mu_{s}+\alpha_{s}\right) \tau} .
$$

Note that $\widetilde{a}_{3}>0$ if $\widetilde{R}_{0}<1$.

Thus, the left-hand side in (17) is positive for all $\lambda \geq 0$ and $\tau>0$ while the right-hand side is zero. This leads to a contradiction. Then, (17) does not have non-negative real solutions. Following the proof of Theorem 1, we know that (17) has eigenvalues with negative real parts when $\tau=0$. Hence, for the case $\tau>0$, if (17) has roots with non-negative real parts they must be complex roots. Moreover, these complex roots should be obtained from a pair of complex conjugate roots crossing the imaginary axis. Thus, (17) must have a pair of purely imaginary roots.

Suppose $\lambda=\omega i(\omega>0)$ is a root of (17). Then, we have

$$
\begin{aligned}
-\omega^{3} i-\tilde{a}_{1} \omega^{2}+\tilde{a}_{2} \omega i+\widehat{a}_{3} \\
=\frac{\xi \rho \Lambda_{s} k_{f}}{\mu_{s}} e^{-\left(\mu_{s}+\alpha_{s}\right) \tau}(\cos (\omega \tau)-i \sin (\omega \tau)),
\end{aligned}
$$

where

$$
\widehat{a}_{3}=\left(\mu_{s}+\alpha_{s}\right) \mu_{p}\left(\mu_{f}+\rho\right) \text {. }
$$

Theorem 3. If $\widetilde{R}_{0}<1$, the disease-free equilibrium $\widetilde{E}_{0}$ of the system (13) is locally asymptotically stable.

Proof. The Jacobian matrix for system (13) is given by

Therefore,

$$
\begin{gathered}
-\omega^{3}+\tilde{a}_{2} \omega=-\frac{\xi \rho \Lambda_{s} k_{f}}{\mu_{s}} e^{-\left(\mu_{s}+\alpha_{s}\right) \tau} \sin (\omega \tau)\left(b_{3}-b_{1} \omega^{2}\right), \\
-\widetilde{a}_{1} \omega^{2}+\widehat{a}_{3}=\frac{\xi \rho \Lambda_{s} k_{f}}{\mu_{s}} e^{-\left(\mu_{s}+\alpha_{s}\right) \tau} \cos (\omega \tau) .
\end{gathered}
$$

From (22), we can get

$$
\left(-\omega^{3}+\widetilde{a}_{2} \omega\right)^{2}+\left(-\widetilde{a}_{1} \omega^{2}+\widehat{a}_{3}\right)^{2}=\left(\frac{\xi \rho \Lambda_{s} k_{f}}{\mu_{s}} e^{-\left(\mu_{s}+\alpha_{s}\right) \tau}\right)^{2}
$$

that is,

$$
\omega^{6}+c_{1} \omega^{4}+c_{2} \omega^{2}+c_{3}=0
$$

Letting $z=\omega^{2}$, we obtain

$$
z^{3}+c_{1} z^{2}+c_{2} z+c_{3}=0
$$

where

$$
\begin{aligned}
c_{1} & =\widetilde{a}_{1}^{2}-2 \widetilde{a}_{2}>0, \\
c_{2} & =\widetilde{a}_{2}^{2}-2 \widetilde{a}_{1} \widehat{a}_{3}>0, \\
c_{3} & =\widehat{a}_{3}^{2}-\left(\frac{\xi \rho \Lambda_{s} k_{f}}{\mu_{s}} e^{-\left(\mu_{s}+\alpha_{s}\right) \tau}\right)^{2} \\
& =\widehat{a}_{3}\left(\widehat{a}_{3}+\frac{\xi \rho \Lambda_{s} k_{f}}{\mu_{s}} e^{-\left(\mu_{s}+\alpha_{s}\right) \tau}\right)\left(1-\widetilde{R}_{0}\right) .
\end{aligned}
$$

If $\widetilde{R}_{0}<1, c_{1}>0, c_{2}>0$, and $c_{3}>0$, which implies that (25) does not have positive solutions, then (24) does not have purely imaginary solutions. Hence, If $\widetilde{R}_{0}<1$, the diseasefree equilibrium $\widetilde{E}_{0}$ of the system (13) is locally asymptotically stable. 
Remark 4. If $R_{0}>1$,

$$
\widetilde{R}_{0}<1 \Longleftrightarrow \tau>\frac{3}{\mu_{s}+\alpha_{s}} \ln R_{0} \triangleq \tau_{0} .
$$

It is easy to see if $R_{0}<1$, we can get $\widetilde{R}_{0}<1$. But if $R_{0}>1$ to get $\widetilde{R}_{0}<1$ the time delay must satisfy $\tau>\tau_{0}$. This implies that to eliminate the disease the time delay must satisfy $\tau>\tau_{0}$. In fact, the time delay is decreasing as the global warming. If the time delay is decreased to be smaller than $\tau_{0}, \widetilde{R}_{0}$ may be larger than 1 , and then the disease may be prevalent.

Now, we turn to the study of the stability of the endemic equilibrium of model (13).

Theorem 5. If $\widetilde{R}_{0}>1$, the endemic equilibrium $\widetilde{E}^{*}$ of the system (13) is locally asymptotically stable.

Proof. The eigenvalues of $\widetilde{E}^{*}$ are $-\mu_{m}$ and the roots of the equation are

$$
\lambda^{4}+b_{1} \lambda^{3}+b_{2} \lambda^{2}+b_{3} \lambda+b_{4}=0
$$

where

$$
\begin{aligned}
b_{1}= & \left(\mu_{s}+\alpha_{s}\right)+\mu_{p}+\left(\mu_{f}+\rho\right)+\left(\mu_{s}+\xi \widetilde{p}^{*}\right)>0, \\
b_{2}= & \left(\mu_{s}+\alpha_{s}\right) \mu_{p}+\left(\mu_{s}+\alpha_{s}\right)\left(\mu_{f}+\rho\right)+\left(\mu_{s}+\alpha_{s}\right)\left(\mu_{s}+\xi \widetilde{p}^{*}\right) \\
& +\mu_{p}\left(\mu_{f}+\rho\right)+\mu_{p}\left(\mu_{s}+\xi \widetilde{p}^{*}\right)+\left(\mu_{f}+\rho\right)\left(\mu_{s}+\xi \widetilde{p}^{*}\right), \\
b_{3}= & \left(\mu_{s}+\alpha_{s}\right) \mu_{p}\left(\mu_{f}+\rho\right)+\left(\mu_{s}+\alpha_{s}\right) \mu_{p}\left(\mu_{s}+\xi \widetilde{p}^{*}\right) \\
& +\mu_{p}\left(\mu_{f}+\rho\right)\left(\mu_{s}+\xi \widetilde{p}^{*}\right)-\xi \rho k_{f} \widetilde{s}^{*} e^{-\left(\mu_{s}+\alpha_{s}\right) \tau} e^{-\lambda}, \\
b_{4}= & \left(\mu_{s}+\alpha_{s}\right) \mu_{p}\left(\mu_{f}+\rho\right)\left(\mu_{s}+\xi \widetilde{p}^{*}\right) \\
& -\xi \rho k_{f} \mu_{s} \widetilde{s}^{*} e^{-\left(\mu_{s}+\alpha_{s}\right) \tau} e^{-\lambda} .
\end{aligned}
$$

Assume that $\lambda \geq 0$. Since $\xi \rho k_{f} \widetilde{s}^{*} e^{-\left(\mu_{s}+\alpha_{s}\right) \tau}=\left(\mu_{s}+\right.$ $\left.\alpha_{s}\right) \mu_{p}\left(\mu_{f}+\rho\right)$ and $0<e^{-\lambda} \leq 1$ for $\tau>0$, we have $b_{1}>0, b_{2}>0$, $b_{3}>0$, and $b_{4}>0$ which lead to a contradiction in (28). Then, (28) does not have non-negative real solutions. From Theorem 2, we know that (28) has solutions with negative real parts for $\tau=0$. Hence, for the case $\tau>0$, if (28) has roots with non-negative real parts they must be complex roots. Moreover these complex roots should be obtained from a pair of complex conjugate roots crossing the imaginary axis. Thus, (28) must have a pair of purely imaginary roots.

Suppose $\lambda=\omega i(\omega>0)$ is a root of (28). Then, we have

$$
\begin{aligned}
& \omega^{4}-b_{1} \omega^{3} i-b_{2} \omega^{2}+\widehat{b}_{3} \omega i+\widehat{b}_{4} \\
& =\xi \rho k_{f} \widetilde{s}^{*} e^{-\left(\mu_{s}+\alpha_{s}\right) \tau}\left(\mu_{s}+\omega i\right)(\cos (\omega \tau)-i \sin (\omega \tau)),
\end{aligned}
$$

where

$$
\begin{aligned}
\widehat{b}_{3}= & \left(\mu_{s}+\alpha_{s}\right) \mu_{p}\left(\mu_{f}+\rho\right)+\left(\mu_{s}+\alpha_{s}\right) \mu_{p}\left(\mu_{s}+\xi \widetilde{p}^{*}\right) \\
& +\mu_{p}\left(\mu_{f}+\rho\right)\left(\mu_{s}+\xi \widetilde{p}^{*}\right), \\
\widehat{b}_{4}= & \left(\mu_{s}+\alpha_{s}\right) \mu_{p}\left(\mu_{f}+\rho\right)\left(\mu_{s}+\xi \widetilde{p}^{*}\right) .
\end{aligned}
$$

Therefore,

$$
\begin{aligned}
& \omega^{4}-b_{2} \omega^{2}+\widehat{b}_{4} \\
& =\xi \rho k_{f} \widetilde{s}^{*} e^{-\left(\mu_{s}+\alpha_{s}\right) \tau}\left(\mu_{s} \cos (\omega \tau)+\omega \sin (\omega \tau)\right), \\
& -b_{1} \omega^{3}+\widehat{b}_{3} \omega \\
& =\xi \rho k_{f} \widetilde{s}^{*} e^{-\left(\mu_{s}+\alpha_{s}\right) \tau}\left(\omega \cos (\omega \tau)-\mu_{s} \sin (\omega \tau)\right) .
\end{aligned}
$$

From (32), we can get

$$
\begin{array}{r}
\left(\omega^{4}-b_{2} \omega^{2}+\widehat{b}_{4}\right)^{2}+\left(-b_{1} \omega^{3}+\widehat{b}_{3} \omega\right)^{2} \\
=\left(\xi \rho k_{f} \widetilde{s}^{*} e^{-\left(\mu_{s}+\alpha_{s}\right) \tau}\right)^{2}\left(\mu_{s}^{2}+\omega^{2}\right) .
\end{array}
$$

Letting $z=\omega^{2}$ again, we obtain

$$
z^{4}+c_{1} z^{3}+c_{2} z^{2}+c_{3} z+c_{4}=0
$$

where

$$
\begin{aligned}
& c_{1}=b_{1}^{2}-2 b_{2}, \\
& c_{2}=b_{2}^{2}+2 \widehat{b}_{4}-2 b_{1} \widehat{b}_{3}, \\
& c_{3}=\widehat{b}_{3}^{2}-2 b_{2} \widehat{b}_{4}-\left(\xi \rho k_{f} s^{*} e^{-\left(\mu_{s}+\alpha_{s}\right) \tau}\right)^{2}, \\
& c_{4}=\widehat{b}_{4}^{2}-\left(\xi \rho k_{f} s^{*} e^{-\left(\mu_{s}+\alpha_{s}\right) \tau}\right)^{2} \mu_{s}^{2} .
\end{aligned}
$$

By similar calculations, we can get $c_{1}>0, c_{2}>0, c_{3}>0$, and $c_{4}>0$. Thus, (34) does not have positive roots and then (28) cannot have purely imaginary solutions. Hence, If $\widetilde{R}_{0}>1$ the endemic equilibrium $\widetilde{E}^{*}$ of the system (13) is locally asymptotically stable.

\section{Simulation and Sensitivity Analysis}

In this section, we perform some computational simulations to observe the impact of the time delay on schistosomiasis dynamics. Through sensitivity analysis of the death rate of snails, the death rate of single parasites, and the death rate of pairs, we give preferable control strategies.

The parameters are chosen with $k_{f}=100$ per year, $k_{m}=$ 145 per year $[13,18], \Lambda_{s}=150$ per year, $\xi=0.000018$ per year [18], $\mu_{s}=0.1$ per year, $\alpha_{s}=0.5$ per year [29], $\mu_{f}=0.2$ per year, $\mu_{m}=0.1$ per year, $\mu_{p}=0.02$ per year, $\rho=0.467$ [9] per year and $\tau=18 / 365 \sim 38 / 365 \approx 0.04 \sim 0.1$ per year [ 4 ]. Then $R_{0}=12.55, \widetilde{R}_{0}=149.9$ and $\tau_{0}=8.43$ when we choose $\tau=30 / 365=0.082$. 
By Remark 4, the time delay $\tau=0.082<8.43=\tau_{0}$ and $\widetilde{R}_{0}>1$ implies that the disease will be prevalent. From Figure 1 we can find, the number of male schistosomes and infected snails increases as the time delay decreases. These phenomena imply that schistosomiasis infection becomes serious as the time delay decreases. As we know, the temperature of snails changes as the environmental temperature changes. Then the environmental temperature can influence the incubation period in infected snails. Currently, the environmental temperature is increasing because of the global warming. Hence, the global warming may reduce the incubation period of infected snails. Consequently, schistosomiasis may become more serious because of the global warming. Hence, the impact of global warming and the incubation period of infected snails on schistosomiasis dynamics can not be ignored.

As for schistosomiasis control, we can control the disease by killing schistosoma and (or) snails. In reality, there are many drugs for killing schistosoma. When we treat definitive hosts with chemotherapy, different drugs aim at different parasites. For example, male schistosoma is more sensitive to praziquantel (PZQ) [30, 31], but pair schistosoma is more sensitive to artesunate (ART) [32]. Hence, sometimes the death rate of pairs and single schistosoma is increased under some treatments, but the extent of increase is different. Under the treatment of some drugs more pairs are killed, but under the treatment of another drugs, more single schistosomes are killed. However, it is a pity that we lack the data about the rate of killing schistosoma to numerical simulation. Hence, we have done some qualitative analyses from the perspective of sensitivity.

Denote by $\varepsilon_{m}, \varepsilon_{f}, \varepsilon_{p}$ and $\varepsilon_{s}$ the killing rates of male schistosoma, female schistosoma, paired schistosoma and snails, respectively. We modify the model by adding these killing rates with the following form:

$$
\begin{aligned}
\frac{d m}{d t} & =k_{m} i(t)-\left(\mu_{m}+\varepsilon_{m}\right) m(t)-\rho f(t), \\
\frac{d f}{d t} & =k_{f} i(t)-\left(\mu_{f}+\varepsilon_{f}\right) f(t)-\rho f(t), \\
\frac{d p}{d t} & =\rho f(t)-\left(\mu_{p}+\varepsilon_{p}\right) p(t), \\
\frac{d s}{d t} & =\Lambda_{s}-\left(\mu_{s}+\varepsilon_{s}\right) s(t)-\xi p(t) s(t), \\
\frac{d i}{d t} & =\xi p(t) s(t)-\left(\mu_{s}+\varepsilon_{s}+\alpha_{s}\right) i(t) .
\end{aligned}
$$

For convenience of computation, we denote

$$
\begin{array}{cc}
\mu_{m}+\varepsilon_{m}=\mu_{m \varepsilon}, & \mu_{f}+\varepsilon_{f}=\mu_{f \varepsilon}, \\
\mu_{p}+\varepsilon_{p}=\mu_{p \varepsilon}, & \mu_{s}+\varepsilon_{s}=\mu_{s \varepsilon} .
\end{array}
$$

Similarly, we can obtain that the basic reproduction number for model (36) is as follows

$$
R_{0 \varepsilon}=\sqrt[3]{\frac{\xi \rho \Lambda_{s} k_{f}}{\mu_{s \varepsilon}\left(\mu_{s \varepsilon}+\alpha_{s}\right) \mu_{p \varepsilon}\left(\mu_{f \varepsilon}+\rho\right)}} .
$$

Note that $R_{0 \varepsilon}$ is independent of the death rate of single male schistosoma. It is easy to see that $R_{0 \varepsilon} \leq R_{0}$, which implies that killing schistosoma and (or) snails can reduce the basic reproduction number and consequently control the disease if $R_{0 \varepsilon}<1$. However, we should choose a rather effective control measure in reality. Thus, we perform some sensitivity analyses by comparing the flexibilities of killing rates. The flexibilities of $\mu_{s \varepsilon}, \mu_{f \varepsilon}$, and $\mu_{p \varepsilon}$ on the basic reproduction number $R_{0 \varepsilon}$, respectively, are

$$
\begin{gathered}
\frac{E R_{0 \varepsilon}}{E \mu_{s \varepsilon}}=\frac{\partial R_{0 \varepsilon}}{\partial \mu_{s \varepsilon}} \times \frac{\mu_{s \varepsilon}}{R_{0 \varepsilon}}=-\frac{1}{3} \frac{2 \mu_{s \varepsilon}+\alpha_{s}}{\mu_{s \varepsilon}+\alpha_{s}}, \\
\frac{E R_{0 \varepsilon}}{E \mu_{f \varepsilon}}=\frac{\partial R_{0 \varepsilon}}{\partial \mu_{f \varepsilon}} \times \frac{\mu_{f \varepsilon}}{R_{0 \varepsilon}}=-\frac{1}{3} \frac{\mu_{f \varepsilon}}{\mu_{f \varepsilon}+\rho}, \\
\frac{E R_{0 \varepsilon}}{E \mu_{p \varepsilon}}=\frac{\partial R_{0 \varepsilon}}{\partial \mu_{p \varepsilon}} \times \frac{\mu_{p \varepsilon}}{R_{0 \varepsilon}}=-\frac{1}{3} .
\end{gathered}
$$

It is easy to see that

$$
\left|\frac{E R_{0 \varepsilon}}{E \mu_{f \varepsilon}}\right|<\frac{1}{3}=\left|\frac{E R_{0 \varepsilon}}{E \mu_{p \varepsilon}}\right|<\left|\frac{E R_{0 \varepsilon}}{E \mu_{s \varepsilon}}\right| .
$$

It implies that the death rate of snails is the most sensitive parameter to the reduction of $R_{0 \varepsilon}$. In addition, the death rate of pair schistosoma is more sensitive than that of female single schistosoma. This is reasonable because the offspring is reproduced by pair schistosoma and the pair schistosoma has stronger effect on the life cycle of schistosome. Hence, to kill the sail hosts may be the first reasonable control measure. Furthermore, to kill pair schistosoma by using some medications may be the other better control measure.

\section{Discussion}

In this paper, we established a new schistosomiasis model including a more reasonable sex ratio of schistosoma, snail dynamics, the latent period of infected snails and mating structure. By choosing the minimum function as the mating function, we studied the stabilities of model (6) without time delay and model (13) with time delay. If the basic reproduction number of model (6) is less than 1, one can prove the stability of the disease-free equilibrium in system (6). When the basic reproductive number $R_{0}$ is greater than 1 , the stability of the endemic equilibrium in system (6) can be obtained. But to get the stability of the disease-free equilibrium in system (13) the time delay must be larger than $\tau_{0}$. In reality, the environmental temperature because of the global warming is increasing. And then the incubation period of infected snails is shortened as the environmental temperature increases [13]. From Figure 1 we know that schistosomiasis infection become more serious as the incubation period of infected snails decreases. Hence, the impact of the incubation period of infected snails during global warming on schistosomiasis transmission cannot be ignored.

In recent years, the control of schistosomiasis remains one of the highest priorities in parasitology. There are many strategies to control schistosomiasis, such as schistosome 


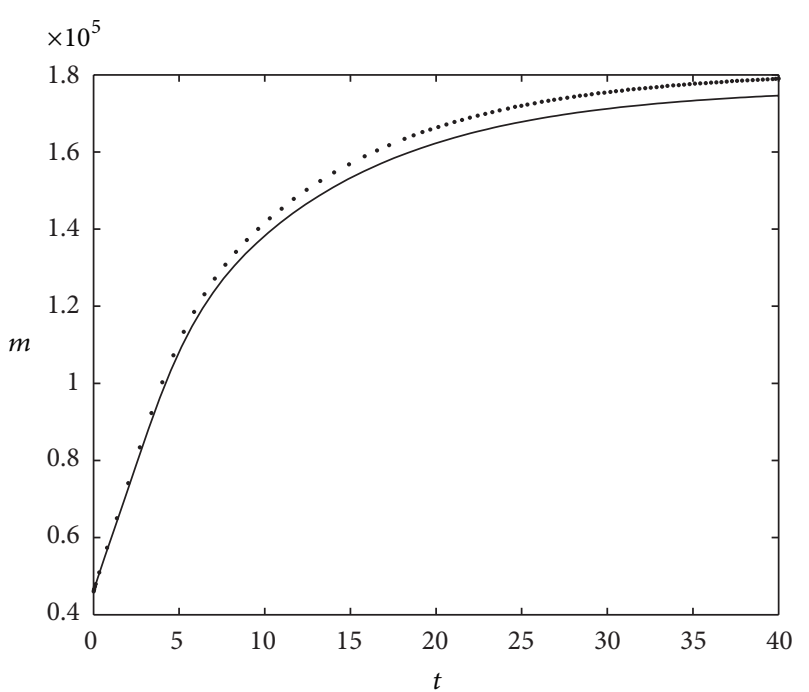

(a) The trajectory of $m$

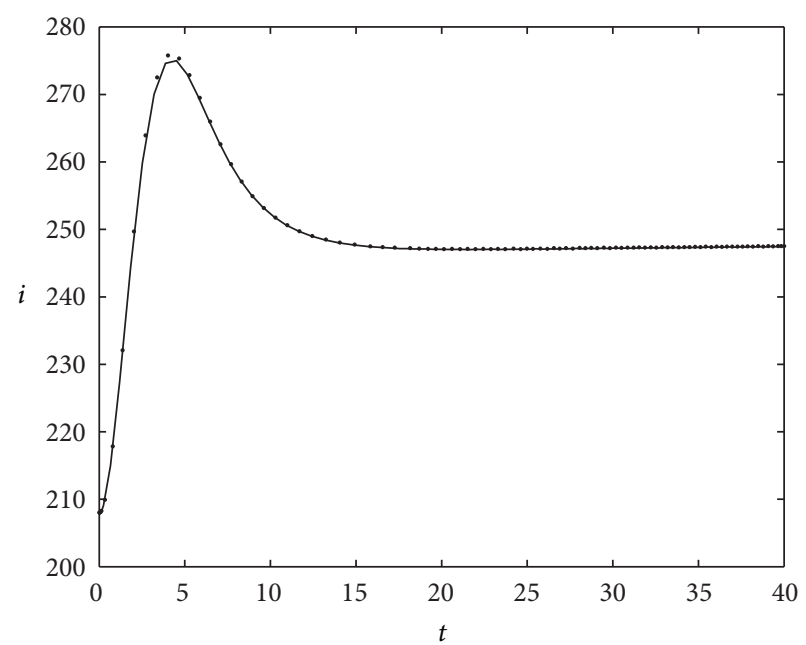

(b) The trajectory of $i$

FIgURE 1: The dot line represents $\tau=0.041$, and the real line represents $\tau=0.082$.

vaccine, killing snails and chemotherapy with PZQ. In this paper, through comparing the flexibilities of $\mu_{s \varepsilon}, \mu_{f \varepsilon}$, and $\mu_{p \varepsilon}$ on $R_{0 \varepsilon}$, we know that the death rate of snails $\mu_{s \varepsilon}$ is the most sensitive to the reduction of $R_{0 \varepsilon}$. Hence killing the snail hosts may be the most reasonable control measure.

Our sensitivity analysis also deduces that $R_{0 \varepsilon}$ is more sensitive to the death rate of pair parasites than to the death rate of female parasite. This implies that killing paired schistosoma is more advantageous than killing single schistosoma to control disease. From the perspective of medicine, eggs are generally accepted as the major cause of pathogenesis of schistosomiasis [14, 30-32]. Nino Incani et al. [14] suggest that the eggs produced by paired schistosoma are the pivotal factor in nosogenesis and transmission, and sexual maturation is the precondition of producing eggs. This means that paired schistosoma is mostly harmful to definitive hosts. Controlling schistosomiasis must attack the ability of the paired schistosoma to produce eggs. Hence, the drug of choice is possibly sufficient for reducing egg-associated pathology.

As we know, PZQ is currently considered the drug of choice for the treatment of schistosomiasis because of its efficacy against all schistosome species, lack of serious side effects, and low cost. However, PZQ has its limitation. PZQ was found to be more active against single male schistosoma $[30,31]$, not paired schistosomes. In paired schistosoma, male schistosoma are folded together with female schistosoma. The egg production is owed to the paired schistosomes. Reducing male schistosoma in paired schistosoma can help reduce the egg production and consequently the overall parasite density and disease prevalence. In additional, from our analysis, $R_{0 \varepsilon}$ is sensitive to the death rate of pair schistosoma and independent of the death rate of single male schistosoma. Furthermore, recent epidemiological evidence suggests the emergence of PZQ-resistant schistosoma [33]. Hence, PZQ may not be the best drug for schistosomiasis.
Currently, there are some drugs which are possibly sufficient for reducing egg-associated pathology. For example, ART significantly decreased the survival time of both paired male and female schistosomes. It might be responsible for damaging reproductive organs or killing schistosoma [32]. Oxamniquine-praziquantel composite in the treatment of schistosoma mansoni infection is also a controlled trial [31].

In summary, although PZQ remains the drug of choice to treat schistosomiasis, it does not protect from reinfection (especially in children) and is minimally effective against larval stages of the parasite. Resistance can also develop, although its mode of action is poorly understood. Therefore an important priority in developing new control strategies is to search new drug targets, in combination with selection of viable vaccine candidates. Based on our analysis in this paper, we can obtain the following two results. Killing the snail hosts may be the most reasonable control measure. If we choose chemotherapy, we should choose some drugs which are sufficient for reducing egg-associated pathology because paired schistosoma is mostly harmful to definitive hosts.

\section{Acknowledgments}

This research is supported by the National Natural Science Foundation of China (11126177 and 11071011), the Natural Science Foundation of Anhui Province (1208085QA15), the Foundation for Young Talents in College of Anhui Province (2012SQRL021), the National Scholarship Foundation of China, Funding Project for Academic Human Resources Development in Institutions of Higher Learning under the Jurisdiction of Beijing Municipality (PHR201107123), the Key Natural Science Foundation of the Anhui Higher Education Institutions of China (KJ2009A49), and the Doctoral Fund of Ministry of Education of China (20113401110001 and 20103401120002). 


\section{References}

[1] E. C. Faust and H. E. Meleney, "Studies on Schistosomiasis japonica," American Journal of Hygiene, no. 3, 1924.

[2] C. P. de Souza, L. K. Jannotti-Passos, S. S. Ferreira, and I. B. Sampaio, "Influence of temperature on development of Schistosoma mansoni female cercariae in Biomphalaria glabrata," Revista do Instituto de Medicina Tropical de São Paulo, vol. 37, no. 4, pp. 319-324, 1995.

[3] A. Perlowagora-Szumlewicz, "The reaction of Australorbis glabratus (Biomphalaria glabrata) to infection with Schistosoma mansoni," Revista do Instituto de Medicina Tropical de Sao Paulo, vol. 10, no. 4, pp. 219-228, 1968.

[4] C. Castillo-Chavez, Z. Feng, and D. Xu, "A schistosomiasis model with mating structure and time delay," Mathematical Biosciences, vol. 211, no. 2, pp. 333-341, 2008.

[5] K. P. Hadeler, R. Waldstätter, and A. Wörz-Busekros, "Models for pair formation in bisexual populations," Journal of Mathematical Biology, vol. 26, no. 6, pp. 635-649, 1988.

[6] S. F. H. Schmitz and C. Castillo-Chavez, "A note on pairformation functions," Mathematical and Computer Modelling, vol. 31, no. 4-5, pp. 83-91, 2000.

[7] L. Qi, J. A. Cui, Y. Gao, and H. Zhu, "Modeling the schistosomiasis on the islets in Nanjing," International Journal of Biomathematics, vol. 5, no. 4, Article ID 1250037, 17 pages, 2012.

[8] L. X. Qi and J. A. Cui, "A schistosomiasis model with praziquantel resistance," Discrete Dynamics in Nature and Society, vol. 2013, Article ID 945767, 13 pages, 2013.

[9] D. Xu, J. Curtis, Z. Feng, and D. J. Minchella, "On the role of schistosome mating structure in the maintenance of drug resistant strains," Bulletin of Mathematical Biology, vol. 67, no. 6, pp. 1207-1226, 2005.

[10] J. Boissier and H. Mone, "Experimental observations on the sex ratio of adult Schistosoma mansoni, with comments on the natural male bias," Parasitology, vol. 121, no. 4, pp. 379-383, 2000.

[11] V. Haukisalmi, H. Henttonen, and P. Vikman, "Variability of sex ratio, mating probability and egg production in an intestinal nematode in its fluctuating host population," International Journal for Parasitology, vol. 26, no. 7, pp. 755-763, 1996.

[12] J. D. Liberatos, "Schistosoma mansoni: male-biased sex ratios in snails and mice," Experimental Parasitology, vol. 64, no. 2, pp. 165-177, 1987.

[13] S. B. Mao, Biology of Schistosome and Control of Schistosomiasis, People's Health Press, Beijing, China, 1990 (Chinese).

[14] R. Nino Incani, G. Morales, and I. M. Cesari, "Parasite and vertebrate host genetic heterogeneity determine the outcome of infection by Schistosoma mansoni," Parasitology Research, vol. 87, no. 2, pp. 131-137, 2001.

[15] S. Rowntree and C. James, "Single sex cercariae of Schistosoma mansoni, a comparison of male and female infectivity," Journal of Helminthology, vol. 51, no. 1, pp. 69-70, 1977.

[16] M. L. Steinauer, I. N. Mwangi, G. M. Maina et al., "Interactions between natural populations of human and rodent schistosomes in the lake victoria region of Kenya: a molecular epidemiological approach," PLoS Neglected Tropical Diseases, vol. 2, no. 4, article e222, 2008.

[17] I. Nåsell, "Mating models for schistosomes," Journal of Mathematical Biology, vol. 6, no. 1, pp. 21-35, 1978.

[18] P. Zhang, G. J. Sandland, Z. Feng, D. Xu, and D. J. Minchella, "Evolutionary implications for interactions between multiple strains of host and parasite," Journal of Theoretical Biology, vol. 248, no. 2, pp. 225-240, 2007.

[19] M. L. Steinauer, "The sex lives of parasites: investigating the mating system and mechanisms of sexual selection of the human pathogen Schistosoma mansoni," International Journal for Parasitology, vol. 39, no. 10, pp. 1157-1163, 2009.

[20] R. M. May and M. E. J. Woolhouse, "Biased sex ratios and parasite mating probabilities," Parasitology, vol. 107, no. 3, pp. 287-295, 1993.

[21] L. A. T. Tchuente, V. R. Southgate, D. Imbert-Establet, and J. Jourdane, "Change of mate and mating competition between males of Schistosoma intercalatum and S. mansoni," Parasitology, vol. 110, no. 1, pp. 45-52, 1995.

[22] O. D. Standen, “The relationship of sex in Schistosoma mansoni to migration within the hepatic portal system of experimentally infected mice," Annals of Tropical Medicine and Parasitology, vol. 47, pp. 139-145, 1953.

[23] E. M. Cornford and M. E. Huot, "Glucose transfer from male to female schistosomes," Science, vol. 213, no. 4513, pp. 1269-1271, 1981.

[24] E. M. Cornford and A. M. Fitzpatrick, "Glucose transfer from male to female schistosomes: definition of the mechanism and rate constant," Molecular and Biochemical Parasitology, vol. 17, no. 2, pp. 131-141, 1985.

[25] E. M. Cornford, "Influence of mating on surface nutrient exchange in schistosomes," Journal of Chemical Ecology, vol. 12, no. 8, pp. 1777-1796, 1986.

[26] B. G. Atkinson and K. H. Atkinson, "Schistosoma mansoni: oneand two-dimensional electrophoresis of proteins synthesized in vitro by males, females, and juveniles," Experimental Parasitology, vol. 53, no. 1, pp. 26-38, 1982.

[27] A. H. Davis, R. Blanton, and P. Klich, "Stage and sex specific differences in actin gene expression in Schistosoma mansoni," Molecular and Biochemical Parasitology, vol. 17, no. 3, pp. 289298, 1985.

[28] P. van den Driessche and J. Watmough, "Reproduction numbers and sub-threshold endemic equilibria for compartmental models of disease transmission," Mathematical Biosciences, vol. 180, pp. 29-48, 2002.

[29] Z. Feng, J. Curtis, and D. J. Minchella, "The influence of drug treatment on the maintenance of schistosome genetic diversity," Journal of Mathematical Biology, vol. 43, no. 1, pp. 52-68, 2001.

[30] S. P. Da Silva and F. Noel, "Time course of the effect of praziquantel on Schistosoma mansoni attachment in vitro: comparison with its effects on worm length and motility," Parasitology Research, vol. 81, no. 7, pp. 543-548, 1995.

[31] M. L. A. Ferrari, P. M. Z. Coelho, C. M. F. Antunes, C. A. P. Tavares, and A. S. Da Cunha, "Efficacy of oxamniquine and praziquantel in the treatment of Schistosoma mansoni infection: a controlled trial," Bulletin of the World Health Organization, vol. 81, no. 3, pp. 190-196, 2003.

[32] Y. Mitsui, M. Miura, and Y. Aoki, "In vitro effects of artesunate on the survival of worm pairs and egg production of Schistosoma mansoni," Journal of Helminthology, vol. 83, no. 1, pp. 7-11, 2009.

[33] D. Cioli, "Chemotherapy of schistosomiasis: an update," Parasitology Today, vol. 14, no. 10, pp. 418-422, 1998. 


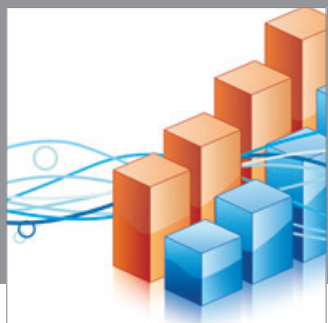

Advances in

Operations Research

mansans

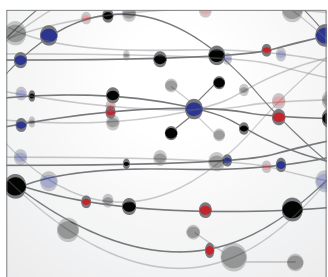

The Scientific World Journal
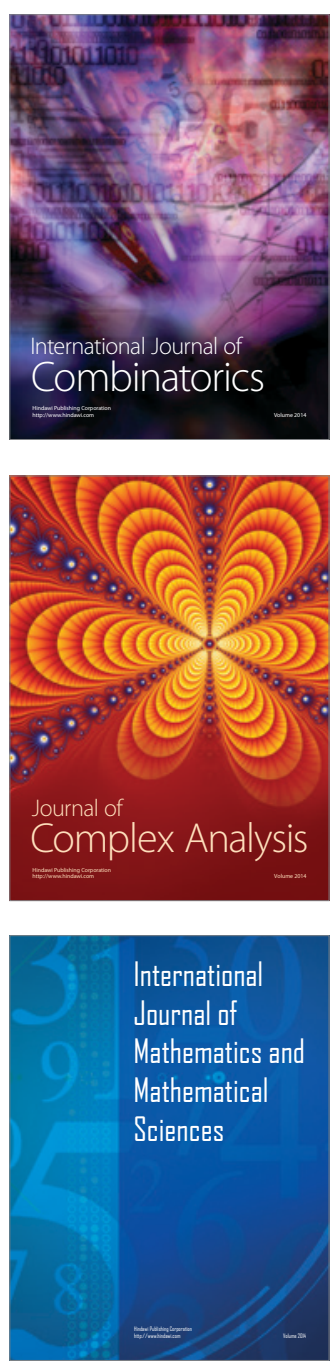
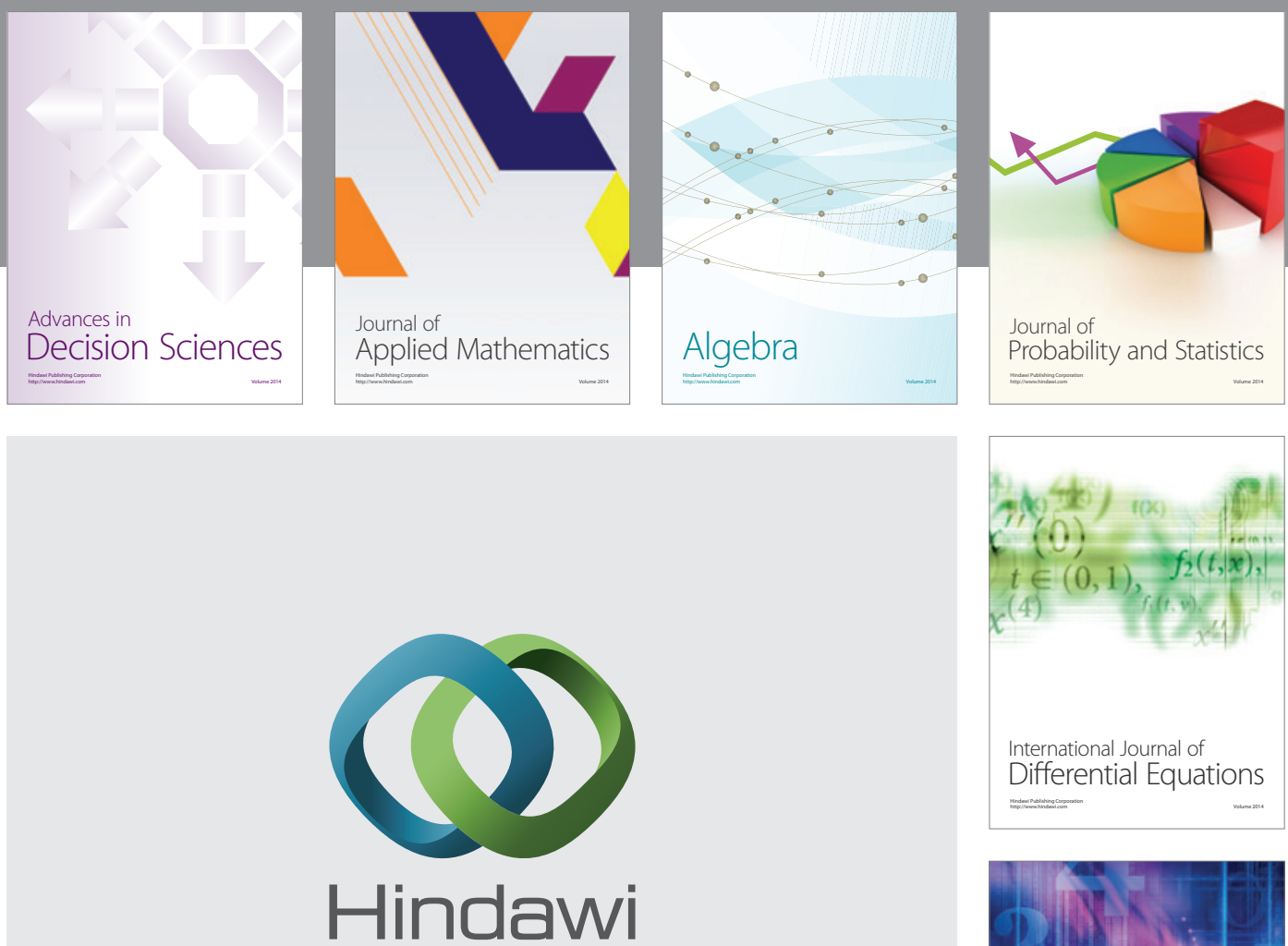

Submit your manuscripts at http://www.hindawi.com
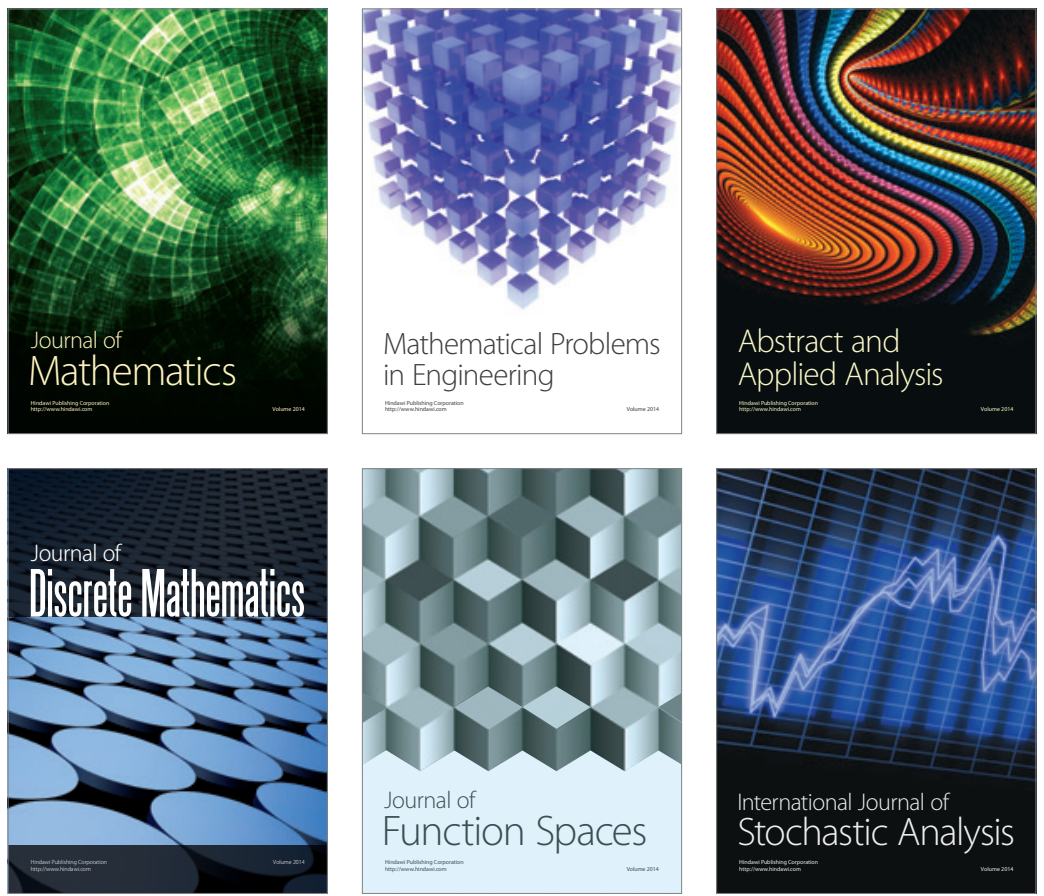

Journal of

Function Spaces

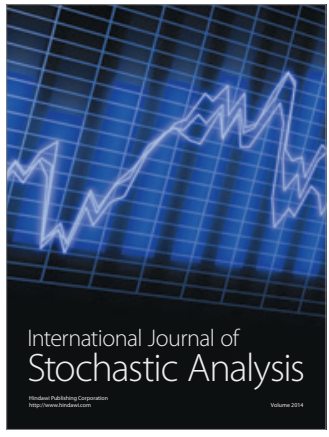

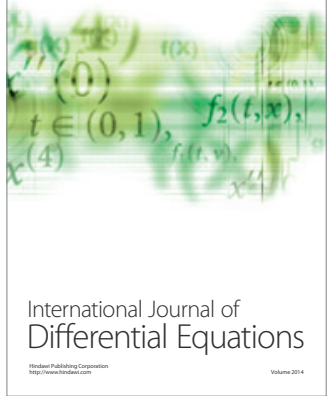
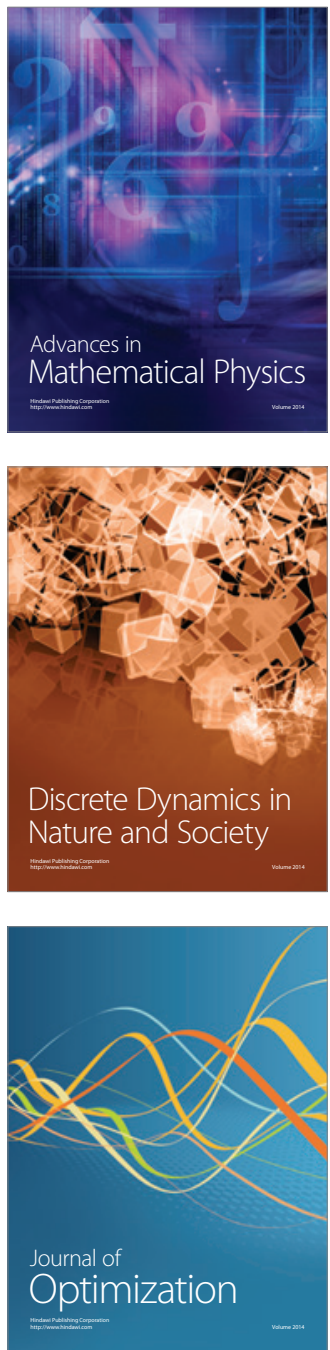